\title{
INCREASED RECOMBINATION AND SELECTION IN BARLEY POPULATIONS CARRYING A MALE STERILITY FACTOR \\ II. GENOTYPIC FREQUENCIES AT MARKER LOCI
}

\author{
S. K. JAIN
}

Department of Agronomy and Range Science, University of California, Davis

Received 18.xi.68

\section{INTRODUGTION}

In barley, a predominantly self-pollinating species, populations derived from several composite crosses have provided evidence for heterozygote advantage and optimising selection (Jain and Allard, 1960; other references in Allard, Jain and Workman, 1968). Characteristically, one finds in these materials a great store of variability and a steady increase in agronomic productivity with the advancing generations. In a previous paper, Jain and Suneson (1966) analysed quantitative variation in populations of the so-called "selfing" and " outbreeding" series that involved low and high proportion of male sterile individuals respectively such as to yield two levels of increased outbreeding and recombination rates in the bulk populations. Although, as expected, a proportionate increase was observed in both between- and within-family components of genetic variability in the "outbreeding" series, this was not accompanied by any greater rate of improvement in population fitness (as measured by fecundity and total seed yield). In fact, it appeared that the population fitness of the outbreeding series did not improve at a sufficiently high rate such as to counterbalance the load due to male sterility component and the increased segregational load at heterotic loci. Such a pattern of response and the evidence for optimising selection reported earlier suggests that the non-additive genetic component of variation is large. However, whether heterozygotes at the marked chromosome segments are favoured through a homeostatic model (Lerner, 1954) or an intermediate optimum model (Robertson, 1956), has not yet been verified. In the present paper, data on genotypic frequencies and character associations are reported to examine the effect of increased outbreeding rates on the levels of heterozygosis and the mode of natural selection at "marker" loci which under the present experimental setup represent chromosome segments of variable lengths around the designated loci.

\section{Materials AND methods}

The experimental materials, consisting of six bulk populations derived from Composite Cross XIV, and the plans of experimental plantings were described by Jain and Suneson (1966). Briefly, in the "selfing" series, generations $F_{5}, F_{15}$, and $F_{22}$ were used that involved nearly 19 per cent., 10 per cent. and 1 per cent. male-steriles respectively; in the " outbreeding" series, on the other hand, generations $\mathrm{O}_{7} \mathrm{~F}_{4}, \mathrm{O}_{15} \mathrm{~F}_{4}$ and $\mathrm{O}_{7} \mathrm{~F}_{12}$ were used 


\section{TABLE 1}

Estimation of genotypic frequencies

\begin{tabular}{|c|c|c|c|c|c|c|c|}
\hline \multirow[b]{2}{*}{ Locus } & \multirow[b]{2}{*}{ Genotype } & \multicolumn{6}{|c|}{ Generation } \\
\hline & & $F_{5}$ & $\mathrm{~F}_{15}$ & $\mathrm{~F}_{22}$ & $\mathrm{O}_{7} \mathrm{~F}_{4}$ & $\mathrm{O}_{15} \mathrm{~F}_{4}$ & $\mathrm{O}_{7} \mathrm{~F}_{12}$ \\
\hline \multirow[t]{3}{*}{$m s^{*}$} & $D$ & .568 & .896 & .948 & $\cdot 688$ & .885 & .819 \\
\hline & $H$ & $\cdot 246$ & .093 & .042 & $\cdot 312$ & $\cdot 115$ & $\cdot 181$ \\
\hline & $R$ & & & & - & - & 一 \\
\hline \multirow[t]{3}{*}{$r$} & $D$ & .579 & $\cdot 610$ & .692 & .521 & .542 & .706 \\
\hline & $H$ & $\cdot 188$ & $\cdot 125$ & $\cdot 105$ & $\cdot 156$ & $\cdot 154$ & .076 \\
\hline & $R$ & $\cdot 233$ & $\cdot 265$ & $\cdot 203$ & $\cdot 323$ & $\cdot 304$ & $\cdot 218$ \\
\hline \multirow[t]{3}{*}{$b 1 \dagger$} & $D$ & $\cdot 185$ & $\cdot 123$ & $\cdot 168$ & $\cdot 104$ & $\cdot 135$ & $\cdot 164$ \\
\hline & $H$ & $\cdot 159$ & .096 & $\cdot 094$ & $\cdot 244$ & $\cdot 135$ & $\cdot 113$ \\
\hline & $R$ & .656 & .781 & .738 & .652 & .730 & .723 \\
\hline \multirow[t]{3}{*}{$e$} & $D$ & $\cdot 146$ & $\cdot 188$ & $\cdot 142$ & $\cdot 137$ & $\cdot 114$ & .087 \\
\hline & $H$ & $\cdot 062$ & $\cdot 052$ & $\cdot 032$ & .095 & .032 & $\cdot 011$ \\
\hline & $R$ & .792 & .760 & .826 & .768 & $\cdot 8 \tilde{5} 4$ & .902 \\
\hline \multirow[t]{3}{*}{$s$} & $D$ & $\cdot 167$ & $\cdot 188$ & $\cdot 137$ & $\cdot 208$ & $\cdot 156$ & $\cdot 128$ \\
\hline & $H$ & .075 & .021 & $\cdot 010$ & $\cdot 114$ & .062 & .053 \\
\hline & $R$ & .758 & $\cdot 791$ & $\cdot 853$ & .678 & .782 & .819 \\
\hline \multirow[t]{3}{*}{$g$} & $D$ & $\cdot 302$ & $\cdot 322$ & $\cdot 326$ & $\cdot 229$ & $\cdot 385$ & $\cdot 415$ \\
\hline & $H$ & $\cdot 125$ & $\cdot 073$ & $\cdot 053$ & $\cdot 114$ & .084 & .064 \\
\hline & $R$ & .573 & .605 & .621 & $\cdot 657$ & $\cdot 531$ & .521 \\
\hline \multirow[t]{3}{*}{$y$} & $D$ & .010 & $\cdot 010$ & .011 & $\cdot 010$ & 0 & .011 \\
\hline & $H$ & .031 & .010 & 0 & $\cdot 042$ & 0 & .044 \\
\hline & $R$ & .959 & .980 & •989 & $\cdot 948$ & 1.000 & .945 \\
\hline \multirow[t]{3}{*}{ pau } & $D$ & .455 & .465 & .417 & $\cdot 467$ & .315 & .406 \\
\hline & $H$ & $\cdot 207$ & .070 & .083 & $\cdot 107$ & $\cdot 162$ & $\cdot 109$ \\
\hline & $R$ & .338 & .465 & .500 & .426 & .523 & .485 \\
\hline
\end{tabular}

* In outbreeding series, only male-fertile spikes were used in progeny tests.

$\dagger$ Genotypic frequency estimated both from the parental and progeny samples due to xenia. These values represent average over both loci $b 1_{1}$ and $b 1_{2}$.

TABLE 2

Generation means for polymorphic index $(P I)$ and heterozygosity $(H)$

$\begin{array}{ccccccc}\text { Quantity } & \mathrm{F}_{5} & \mathrm{~F}_{15} & \mathrm{~F}_{22} & \mathrm{O}_{7} \mathrm{~F}_{4} & \mathrm{O}_{15} \mathrm{~F}_{4} & \mathrm{O}_{7} \mathrm{~F}_{12} \\ \overline{\mathrm{PI}} & \cdot 180 & \cdot 115 & \cdot 140 & \cdot 178 & \cdot 164 & \cdot 158 \\ \overline{\mathrm{H}} & \cdot 137 & .059 & \cdot 053 & \cdot 125 & .090 & \cdot 067\end{array}$

$\overline{\mathrm{PI}}=\Sigma p(1-p) / n$, sum of allelic frequency product over $n$ loci (including $m s$ ). 
where the designation $\mathrm{O}_{i} \mathrm{~F}_{j+1}$ indicates sibbing for $i$ generations (seed harvested from $m s m s$ 은 $\times M s$ - outpollinations) followed by natural selfing for $j$ generations, viz.

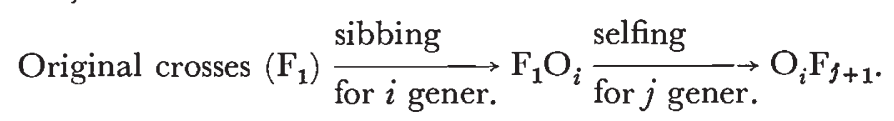

Thus, the outbreeding series involves a pool of half-sib progenies of male steriles pollinated with random $M s$ pollen source and therefore, as high as 45-50 per cent. male sterile segregants in the population. The out-crossing rate $\left(t^{\prime}\right)$ was estimated from the data on outcrosses in the progenies of recessive genotypic classes, frequency of male-steriles ( $m s m s$ ) and the relative seed set on male-steriles. Progeny tests for scoring genotypic frequencies involved spaced planting of a random sample of 200 progenies ( 20 plants per progeny) from each of the six populations. In the case of sibbing series, only the malefertile individuals ( $M s M s, M s m s$ ) were available for progeny tests. The marker loci used in this study were: $r$ (rough $v$ s. smooth awn), b1 (blue $v s$. white aleurone; two complementary factors, $b 1_{1}$ and $b 1_{2}$ were assumed to have identical genotypic frequencies), $e$ (elongate $v s$. short glume awn), $s$ (long $v s$. short rachilla hairs), $g$ (dentate $v s$. nondentate lemma), $v$ (two- $v s$. six-rowed), and pau (purple vs. nonpurple auricle). Loci $e, g$, pau and $v$ have been located on chromosome 2 (recombination values not yet determined unequivocally) and loci $s$ and $r$ are on chromosome 7, with recombination value $\cdot 20$ to $\cdot 25$ (Robertson et al., 1964; Nilan, 1964).

\section{Results}

The estimates of genotypic frequencies $(D, H, R)$ of the dominant, heterozygote and recessive classes at each of the eight marker loci are given in table 1. Based on these data, several comparisons can be made between the genetic composition of the selfing and outbreeding series. In both cases the allelic frequencies $\left(p=1-q=D+\frac{1}{2} H\right)$ shifted slowly in the same direction, with loci $r, b l, g$ and pau remaining highly heterozygous and loci $e$ and $v$ approaching fixation of their recessive alleles. Table 2 gives estimates of the mean polymorphic index $\overrightarrow{(\mathrm{PI})}$ (defined as $\Sigma p_{i} q_{i} / n$, mean allelic product over $n$ loci) and mean heterozygosity $(\overline{\mathrm{H}})$. In $\mathrm{O}_{7} \mathrm{~F}_{12}$ both $\overline{\mathrm{PI}}$ and $\overline{\mathrm{H}}$ are higher than $\mathrm{F}_{22}$ which verifies the maintenance of greater levels of genetic polymorphisms and heterozygosity in the "out-breeding" series. In fact, since only the male-fertile individuals were available for progeny tests in the outbreeding series, this might have resulted in an underestimation of the heterozygote frequencies since the $m s m s$ class, owing to enforced outbreeding, would have contributed proportionately more heterozygotes to the population. The proportion of $m s m s$, as noted earlier, declined from 18 per cent. in $\mathrm{F}_{5}$ to nearly 1 per cent. in $\mathrm{F}_{22}$ whereas in $\mathrm{O}_{7} \mathrm{~F}_{12}$, nearly 6 per cent. ms ms were present; thus, the male-sterile component is retained for a longer period than expected, presumably through the improvement of seed set with advancing generations. Further, it would seem that frequency dependency of a seed set factor $(k)$ allows a low proportion of male-steriles to be retained for long periods, besides its transient role as a factor markedly enhancing outbreeding levels during the early generations (also Jain and Suneson, 1964). 
The outcrossing rates in $\mathrm{F}_{22}$ and $\mathrm{O}_{7} \mathrm{~F}_{12}$, based on the frequency of $m s m s$, the estimates of $k$ directly from the proportion of seed set, and outcrossing rate in the absence of male-steriles assumed to be two per cent., were estimated to be of the order of 0.025 and 0.045 respectively. Assuming that populations in $\mathrm{F}_{22}$ and $\mathrm{O}_{7} \mathrm{~F}_{12}$ have nearly approached equilibrium genotypic frequencies, the selective values are estimated using the models discussed by Workman and Jain (1966). Table 3 gives selective values $(x, y)$ of the dominant and

TABLE 3

Estimates of selective values of homozygotes $(\mathrm{x}, \mathrm{y})^{*}$

\begin{tabular}{|c|c|c|c|c|c|c|c|}
\hline \multirow[b]{2}{*}{ Generation } & \multirow{2}{*}{$\begin{array}{l}\text { Sel. } \\
\text { value }\end{array}$} & \multicolumn{6}{|c|}{ Locus } \\
\hline & & $r$ & $b l$ & $e$ & $s$ & $g$ & pau \\
\hline & $x$ & 0.562 & 0.406 & 0.674 & $1 \cdot 105$ & 0.677 & 0.620 \\
\hline$F_{22}$ & $y$ & 0.521 & 0.506 & 0.689 & 1.098 & 0.685 & 0.624 \\
\hline & & 0.692 & 0.399 & 1.220 & 0.651 & 0.814 & 0.659 \\
\hline $\mathrm{O}_{2} \mathrm{~F}_{12}$ & $y$ & 0.610 & 0.518 & 1.212 & 0.683 & 0.816 & 0.663 \\
\hline
\end{tabular}

* Selective values of dominant, heterozygote, recessive genotypes assumed to be $x: 1: y$; Model II of Workman and Jain (1966) used for locus $b l$, Model I for the others. Mean $t^{\prime}$ was assumed to be 0.025 in $F_{22}$ and 0.045 in $\mathrm{O}_{7} \mathrm{~F}_{12}$ generation, based on the frequency of $m s m s$ in $\mathrm{F}_{22},<1$ per cent., and in $\mathrm{O}_{7} \mathrm{~F}_{12}, 6$ per cent.

recessive homozygotes relative to the heterozygote selective value being unity. Note that there is marked heterozygote advantage at loci $r, b 1$ and pau in both series whereas the values of $(x, y)$ at loci $e$ and $s$ are different in the two series. Thus, the selective forces at certain individual marker loci can vary with the genetic structure and mating system, depending on the role of epistatic interactions, genetic background and the history of population.

Two questions about the role of genetic interactions in these populations are of interest: (1) Are there phenotypic associations between different pairs of loci? Using $2 \times 2$ contingency tables, $\chi_{(1)}^{2}$ for associations for several examples of pairs of loci are given in table 4 . A majority of these values of

TABle 4

Association- $\chi_{(1)}^{2}$ based on $2 \times 2$ contingency tables

\begin{tabular}{lcccc}
\multicolumn{4}{c}{ Generation } \\
\cline { 2 - 5 } Loci & $\mathrm{F}_{\mathrm{b}}$ & $\mathrm{F}_{22}$ & $\mathrm{O}_{7} \mathrm{~F}_{4}$ & $\mathrm{O}_{15} \mathrm{~F}_{4}$ \\
$s, g$ & 3.37 & 1.02 & 0.82 & 1.98 \\
$s, r$ & 9.17 & 0.17 & 0.86 & 0.72 \\
$e$, pau & 0.40 & 3.42 & 1.32 & 1.21 \\
$g$, pau & 2.28 & 0.04 & 0.46 & 0.45 \\
$e, g$ & 0.76 & 2.86 & 0.06 & 0.22
\end{tabular}

$\chi^{2}$ are not significant (at $\mathrm{P}=\cdot 05$ level), and generally the values in the outbreeding series are lower, thus indicating no tendency toward the building up of character associations. Higher recombination rates obtained under the sibbing series perhaps result in too rapid a breakdown of favourable gene complexes. (2) Are heterozygotes at different loci uncorrelated in distribution, or in other words, is there a tendency for cumulative type of heterozygote interactions resulting in an excess of multiple heterozygotes? A 
small amount of excess is expected under partial inbreeding (Haldane, 1949). Fig. 1 gives the frequency distribution of heterozygotes at $0,1,2, \cdots$ loci in the form of histograms; note that in both selfing and outbreeding series, the loss of heterozygosity during the later generations is from the multiple heterozygous classes. The distribution seems to fit the negative binomial or Poisson curves equally well, but clearly there is no tendency for building up of any character associations (as one might expect with epistatic heterotic models, e.g. cumulative overdominance). Optimum-heterotic models, as shown by the other evidence from quantitative data reported earlier and heterozygote advantage at several marker loci, fit the overall picture of

TABLE 5

Estimates of means and variances (based on family means) for genotypes at loci $\mathrm{r}$ and $\mathrm{s}$

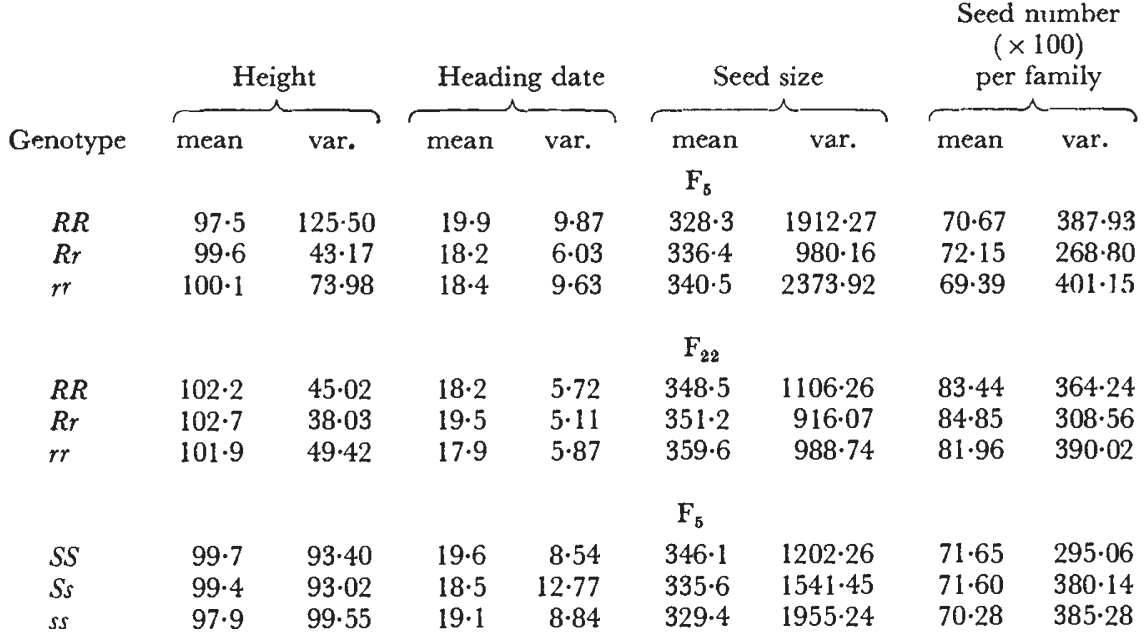

giving no significant associations among the genotypes at various marker loci. This is in contrast to the results from CGII or CGV which involve different base parents and marked pair-wise genotypic frequency associations.

Finally, data on the genotypic constitution and quantitative characters (heading date, height, seed number and seed size) allow us to test the differences among homozygotes and heterozygotes in terms of the mean and relative distribution of family means as given by $\sigma_{B}^{2}$ within each class. To illustrate loci with or without an excess of heterozygote frequencies over expected in the $F_{22}$ generation, say $r$ and $s$, table 5 gives means and variance estimates derived from the family means. In general, there is evidence for heterozygotes being intermediate in mean with a greater mean seed number; further, it is striking to note that heterozygous class has in a majority of comparisons lower variance which may arise both due to genetic homeostasis (constancy of mean expression over genetic backgrounds and environments) as well as due to relatively less developmental flexibility. These data strongly support an optimum model, with heterozygotes being favored for being intermediate phenotypically and thus nearer to the population mean; in case of locus $s$, heterozygotes have intermediate mean fecundity and do not show consistently a lower variance which would under this model lead to the loss of heterozygosis. In general, loci $b 1$ and pau gave similar results to locus $r$ in 


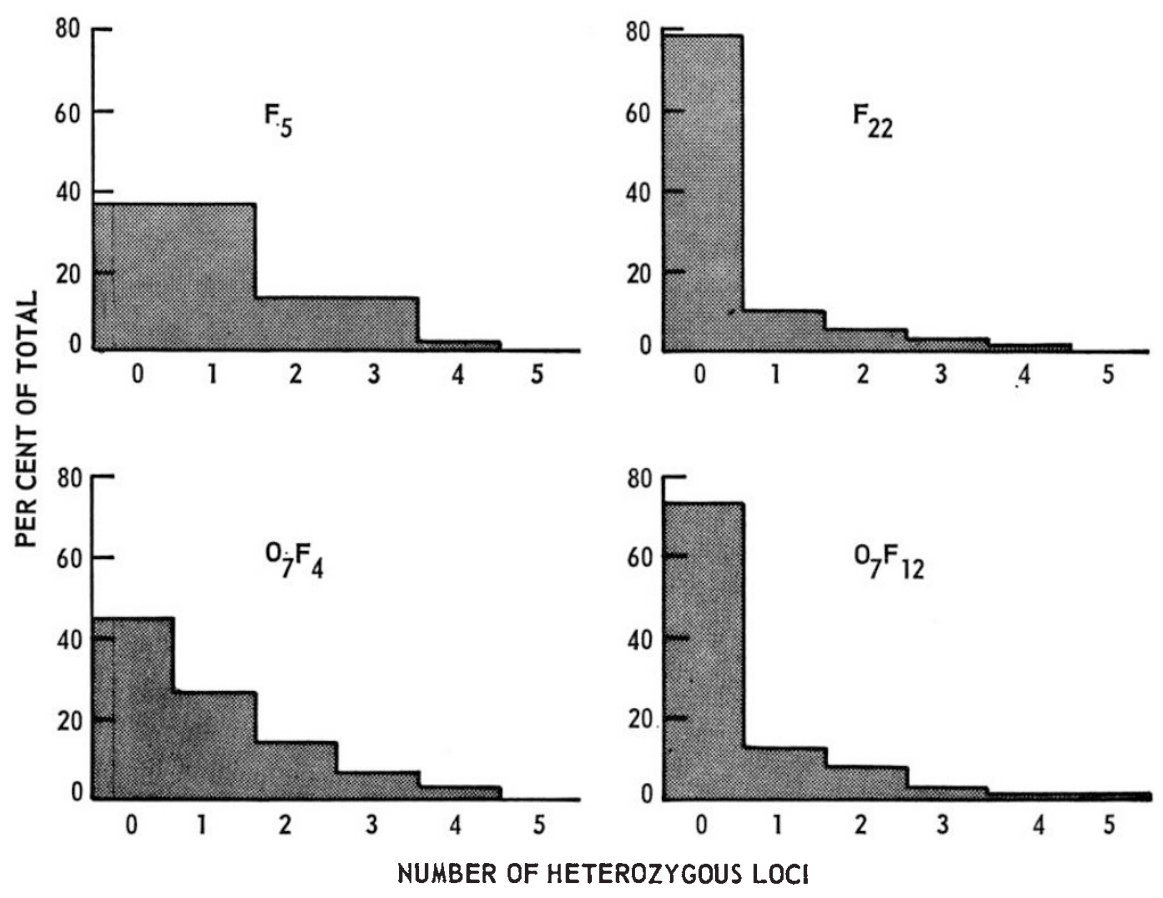

FIG. 1.-Histograms showing the relative proportion of individuals heterozygous at $0,1,2,3$, 4 and 5 loci in $\mathrm{F}_{5}$ vs. $\mathrm{F}_{22}$ and $\mathrm{O}_{7} \mathrm{~F}_{4}$ is. $\mathrm{O}_{7} \mathrm{~F}_{12}$ generations.

both of the series. Thus, outbreeding rate did not influence the basis of heterozygote advantage; in fact, similar results were obtained earlier with CCV populations which did not involve a male sterility factor (Allard, Jain and Workman, 1968, for references).

\section{Discussion}

With the rather limited number of comparisons that these materials allowed in terms of the number of generations available and only two levels of outbreeding, the results, however, clearly showed that the presence of male-steriles enforced relatively higher outbreeding rates, giving a greater level of variation and heterozygosis than the natural selfing series. But as also noted in the case of quantitative genetic changes in fitness or productivity (Jain and Suneson, 1966), the mode of natural selection at marker loci did not seem to be different under increased recombination. Evidence points to the operation of optimum-heterotic model, with no clear indication for the role of cumulative or diminutive models of heterosis; these results further show that the heterozygotes are selectively superior for being phenotypically nearer to the population mean. Robertson's (1956) and Lerner's (1954) models predict the maintenance of genetic variability under such a model, and our recent theoretical work with multigenic models also supports these findings (Jain, 1968a, b). We do not know how stable the observed polymorphisms are since many of these polymorphisms as generated by male 
sterility and consequently, higher levels of outbreeding, might be transient such as to be eventually lost as the generations advance, or as the homozygotes evolve toward better stability under varying environments. It should be noted, however, that selective values $(x, y)$ given above are potent enough to maintain stable polymorphisms even if there are random fluctuations around these values. Relevant to the role of heterosis in stable balanced polymorphisms are the discussions by Spiess (1962), Dobzhansky and Pavlovsky (1959) and Lewontin (1958) among others. Further work has been undertaken to test the effect of frequency dependency (Harding et al., 1967), constant vs. fluctuating environments, ecological interactions, developmental stability and the idea of locus-specific hybridity $v s$. general optimum hybridity ( $c f$. Darlington and Mather, 1949) in inbreeding populations. Note that most of these ideas have been recognised in the literature and discussed by numerous workers dealing with the case of random mating population. Here, again, the differences in genetic structures, adaptive strategies, and the role of heterosis and homeostasis seems to differ only in degree with the mating system (Allard, Jain and Workman, 1968). Attempts to discuss these as basically different, rather than a spectrum of evolutionary materials, are in large part unwarranted so far.

Increased variability under these experimental schemes may not have produced new or greater response under natural selection; however, it is conceivable that artificial selection might yield relatively greater shifts in population performance under mass selection, which would be useful from the breeder's viewpoint. Further studies have now been planned to evaluate certain breeding procedures in these barley populations.

\section{Summary}

1. Six bulk-hybrid populations of barley involving two different levels of outbreeding enforced by the presence of male-steriles (termed as "selfing" and "outbreeding" series) were studied for changes in the genotypic frequencies at eight marker loci.

2. A higher level of heterozygosis and consequently higher polymorphic index were obtained in the outbreeding series, with the general pattern of changes in allelic frequencies and character association being similar in the two series.

3. Comparisons of homozygote and heterozygote classes by their mean and variance of family means for heading date, plant height, seed size and seed number, in general, showed heterozygous families to be favoured for being generally near the population mean and thus suggest the operation of an optimum-heterotic model.

4. These findings confirm our earlier conclusion that the form and intensity of natural selection is well adjusted to the variability system attained under the predominantly selfing system which is not readily modified by the introduction of a male sterility factor.

Acknowledgment.--I am deeply indebted to Mr C. A. Suneson for providing seed materials and basic records on Composite Cross XIV. This work was supported in part by a U.S. Public Health Service grant (GM 10476). 


\section{REFERENCES}

ALlARD, R. W., JAIN, s. K., AND WORKMAN, P. L. 1968. The genetics of inbreeding populations. Advan. Genet., 15, 55-131.

DARlington, c. D., ANd MAther, K. 1949. The Elements of Genetics. Allen and Unwin, London.

DOBZHANSKY, TH., AND PAVLOVSKY, O. 1959. How stable is balanced polymorphism? Proc. Natl. Acad. Sci. U.S., 46, 41-47.

HALDANE, J. B. s. 1949. The association of characters as a result of inbreeding and linkage. Ann. Eugen., 15, 15-23.

HARDing, J., AllaRd, R. W., AND SMELTZeR, D. G. 1967. Population studies in predominantly self-pollinating species. IX. Frequency-dependent selection in Phaseolus lunatus. Proc. Natl. Acad. Sci. U.S., 56, 99-104.

JAIN, s. K. 1968a. Epistasis and linkage in inbreeding populations. Proc. XII Intern. Genetics Congress, Tokyo, 2, 143-144.

JAIN, s. K. 1968b. Simulation of models involving mixed selfing and random mating. II. Selection, linkage and drift in finite populations. Theor. and Appl. Genetics, 38, 232-242.

JAIN, s. K., AND ALLARD, R. w. 1960. Population studies in predominantly self-pollinating species. I. Evidence for heterozygote advantage in a closed population of barley. Proc. Natl. Acad. Sci. U.S., 46, 1371-1377.

JAIN, s. K., AND SUNESON, c. A. 1964. Population studies in predominantly self-pollinating species. VII. Survival of a male-sterility gene in relation to heterozygosis in barley populations. Genetics, 50, 905-913.

JAIN, s. K., AND SUNESON, c. A. 1966. Increased recombination and selection in barley populations carrying a male sterility factor. I. Quantitative variability. Genetics, 54, $1215-1224$.

LERner, I. M. 1954. Genetic Homeostasis. John Wiley and Sons, New York.

LEWONTIN, R. C. 1958. Studies on heterozygosity and homeostasis. II. Loss of heterosis in a constant environment. Evolution, 12, 494-503.

NILAN, R. A. 1964. Cytology and genetics of barley. Monograph Suppl. no. 3. Research Studies. Washington State University, Pullman.

ROBERTSON, A. 1956. The effect of selection against extreme deviants based on deviation or on homozygosis. Four. Genetics, 54, 236-248.

ROBERTSON, D. W., WiEbe, G. A., SHANDS, R. G., AND HAGBerg, A. 1965. A summary of linkage studies in cultivated barley, Hordeum species: Supplement III, 1954-1963. Crop Sci., 5, 33-43.

SPIEss, E. 1962. Papers on Animal Population Genetics: Introduction. Little, Brown and Co., Boston.

WORKMAN, P. L., AND JAIN, s. K. 1966. Zygotic selection under mixed random mating and self-fertilization: Theory and problems of estimation. Genetics, 54, 159-171. 